\title{
Effect of Thermal Cycling on the Structural Evolution of Methylammonium Lead lodide Monitored around the Phase Transition Temperatures
}

\author{
Anastasiia Glushkova,* Konstantins Mantulnikovs, Gaétan Giriat, Konstantin Semeniuk, \\ László Forró, Endre Horváth, and Alla Arakcheeva
}

Optoelectronic devices and solar cells based on organometallic hybrid perovskites have to operate over a broad temperature range, which may contain their structural phase transitions. For instance, the temperature of $330 \mathrm{~K}$, associated with the tetragonal-cubic transformation, may be crossed every day during the operation of solar cells. Therefore, the analysis of thermal cycling effects on structural and electronic properties is of significant importance. This issue is addressed in the case of methylammonium lead iodide $\left(\mathrm{CH}_{3} \mathrm{NH}_{3} \mathrm{Pbl}_{3}\right)$ across both structural phase transitions (at 160 and $330 \mathrm{~K}$ ). In situ synchrotron radiation $\mathrm{X}$-ray diffraction (XRD) data recorded between 140 and $180 \mathrm{~K}$ show the emergence of a boundary phase between the orthorhombic and tetragonal phases, which becomes more abundant with successive thermal cycles. At high temperatures, around $330 \mathrm{~K}$, an incommensurately modulated tetragonal phase is formed upon repeated crossings of the phase boundary between tetragonal and cubic phases. These alterations, which indicate a gradual evolution of the material under operating conditions of photovoltaic devices, are further documented by electrical resistivity and heat capacity measurements.

\section{Introduction}

Methylammonium lead iodide $\left(\mathrm{MAPbI}_{3}\right)$ is an efficient lightabsorbing photovoltaic material, which, due to the abundance of intriguing optoelectronic properties, ${ }^{[1-3]}$ found applications in many areas of technology such as gas sensors, ${ }^{[4]}$ photodetectors, ${ }^{[5,6]}$ light-emitting diodes, ${ }^{[7]}$ and solar cells. ${ }^{[8]}$ Remarkably, in less than a decade, the efficiency of $\mathrm{MAPbI}_{3}$-based solar cells has improved by an order of magnitude to the present level of $22 \%$. $^{[-11]}$

Although the aforementioned trend is very promising, the applications of this compound are currently limited, primarily due to its chemical instability. ${ }^{[12]}$ It has been shown that the efficiency of $\mathrm{MAPbI}_{3}$-based devices and the structure of the material

A. Glushkova, K. Mantulnikovs, Dr. G. Giriat, Dr. K. Semeniuk,

Prof. L. Forró, Dr. E. Horváth, Dr. A. Arakcheeva

Laboratory of Physics of Complex Matter

EPFL

$\mathrm{CH}-1015$ Lausanne, Switzerland

E-mail: anastasiia.glushkova@epfl.ch

The ORCID identification number(s) for the author(s) of this article can be found under https://doi.org/10.1002/solr.201900044.

DOI: 10.1002/solr.201900044 are strongly dependent on the preparation method and storage conditions (temperature, humidity, and atmosphere). ${ }^{[13,14]}$ Moreover, the transformation of the crystal structure of the perovskite during phase transitions modifies its electronic and phononic band structures, affecting the transport properties. The low-temperature $(160 \mathrm{~K})$ and high-temperature (330 K) phase transitions are both driven by the organic cation positional disorder, which increases on heating from 100 to $352 \mathrm{~K} .^{[15]}$ The two corresponding types of disorders can be classified by the behavior of the $\mathrm{C}-\mathrm{N}$ axis of the methylammonium, $\mathrm{CH}_{3} \mathrm{NH}_{3}{ }^{+}$ (MA). The first disorder type is linked to the number of possible orientations that the $\mathrm{C}-\mathrm{N}$ axis can adopt relative to the inorganic framework. The second one concerns the rotation of the organic cation around the crystallographic axes, which vary between the systems of different crystalline symmetries. Although the organic group is not directly involved in the formation of the band structure, the cation influences it by interacting with the inorganic $\mathrm{PbI}_{6}$ octahedron through the rotational degrees of freedom. ${ }^{[16]}$

Under normal operating conditions, the $\mathrm{MAPbI}_{3}$ active photoabsorption layer in a well-encapsulated solar cell can easily heat over $360 \mathrm{~K}$, consequently crossing the high-temperature phase transition at least twice every $24 \mathrm{~h} .{ }^{[13,17]}$ Thus, the lack of understanding of how periodic temperature changes affect $\mathrm{MAPbI}_{3}$ has prevented the industrial implementation of organometallic hybrid perovskite-based devices. This issue has to be addressed through a detailed investigation of the lattice transformations while repeatedly crossing the phase transition temperature.

In this study, we show that after thermal cycling around either phase transition temperature, $\mathrm{MAPbI}_{3}$ does not return to the initial state. Instead, the material approaches an equilibrium state with specific electronic properties for each phase transition. For the low-temperature transition, we found the presence of a new orthorhombic phase, which with temperature sweeps progressively replaces the low-temperature orthorhombic phase. This change correlates with the reduction in photoconductance as a result of thermal cycling. ${ }^{[18]}$ For the high-temperature transition, we discovered an emergence of an incommensurately modulated tetragonal phase, which structure stabilizes with successive 
transitions. This suggests that there is no boundary phase between the cubic and tetragonal regions because both belong to the same incommensurately modulated structure.

\section{Results and Discussion}

\subsection{The Low-Temperature Orthorhombic to Tetragonal Phase Transition}

At the low-temperature transition $(160 \mathrm{~K})$, both tetragonal and orthorhombic phases coexist in a certain temperature range. ${ }^{[19]}$ The coexistence region starts at the temperature of $130 \mathrm{~K}$, below which the orientation of the $\mathrm{C}-\mathrm{N}$ axis of MA relative to the principal orthorhombic axes is restricted to two directions due to hydrogen bonds between the $\mathrm{NH}_{3}$ group and the nearest I atoms, which also prevents the axial rotation of MA. ${ }^{[20]}$ Upon heating above $130 \mathrm{~K}$, the cation starts to adopt $90^{\circ}$-separated orientations, and by $181 \mathrm{~K}$, all four equivalent directions of the tetragonal unit cell are equally preferred. The organic group remains in this disordered state with four possible alignments up to room temperature. In this phase, the organic cation and the $\mathrm{PbI}_{6}$ octahedra are rotated around the $c$-axis in an alternating manner. Such MA order-disorder transformation induces a transition from tilted to nontilted $\mathrm{PbI}_{6}$ octahedron.

\subsubsection{X-Ray Data}

The effect of thermal cycling between 180 and $140 \mathrm{~K}$ on the

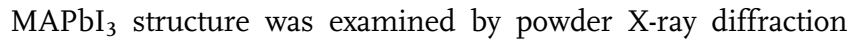
(XRD) experiment (see Experimental Section for details). The XRD profiles obtained during the first cycle cool down and the fourth cycle warm up are shown in Figure 1. The data recorded over four thermal cycles enabled the identification of three distinct crystal structures. Furthermore, the quantitative analysis of the phase composition at a given temperature showed an evolution of the amounts of the different phases in the sample with the number of cycles. For the first and fourth cycles, the temperature dependence of the calculated contribution of the phases and their unit cell volumes are shown in Figure 2, and the unit cell parameters are shown in Figure 3.

First Cycle: Upon cooling from 180 to $140 \mathrm{~K}$, a single tetragonal (Tetr) phase is present down to a temperature of $157 \mathrm{~K}$, below which the orthorhombic (Ort-1) phase appears, as shown in Figure 1a. In accordance with previous reports, ${ }^{[21,22]}$ we observe that both Tetr and Ort- 1 phases coexist down to $140 \mathrm{~K}$. This is illustrated by the XRD profile at $140.89 \mathrm{~K}$ in Figure 1c. The temperature dependence of each phase contribution, which is shown in Figure $2 \mathrm{a}$ with the coefficients $k_{1}$ for the Tetr and $k_{2}$ for the Ort-1 phase, indicates an equal contribution at $142 \mathrm{~K}$. When (a)

(b)
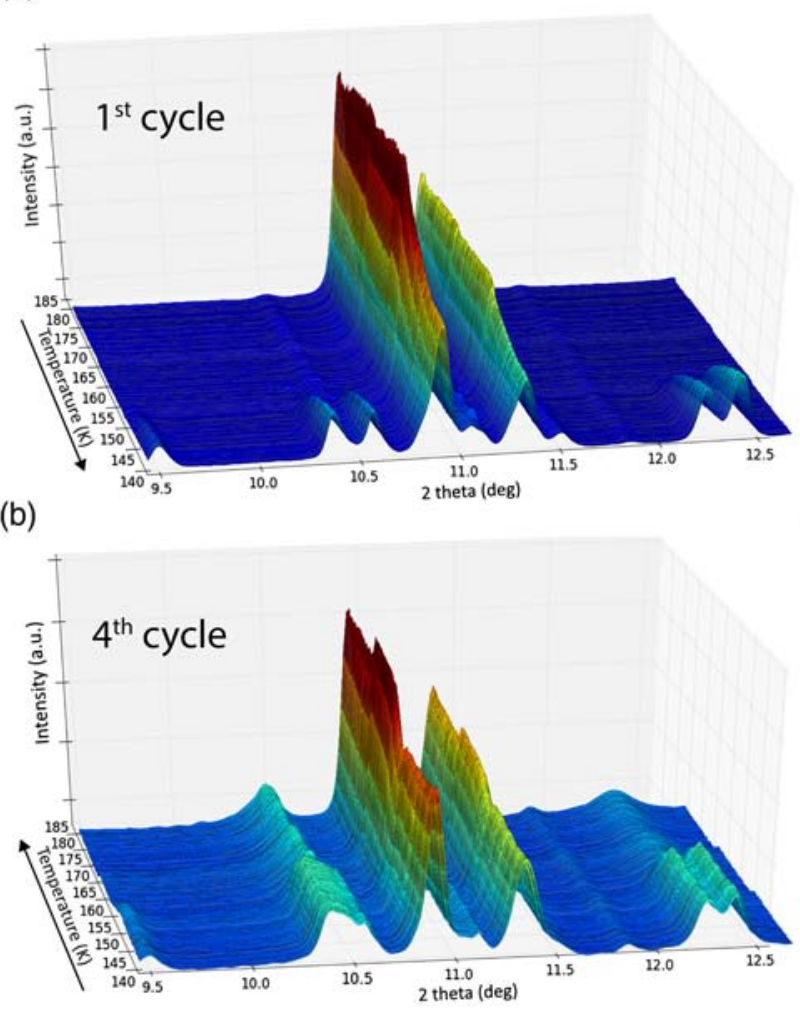
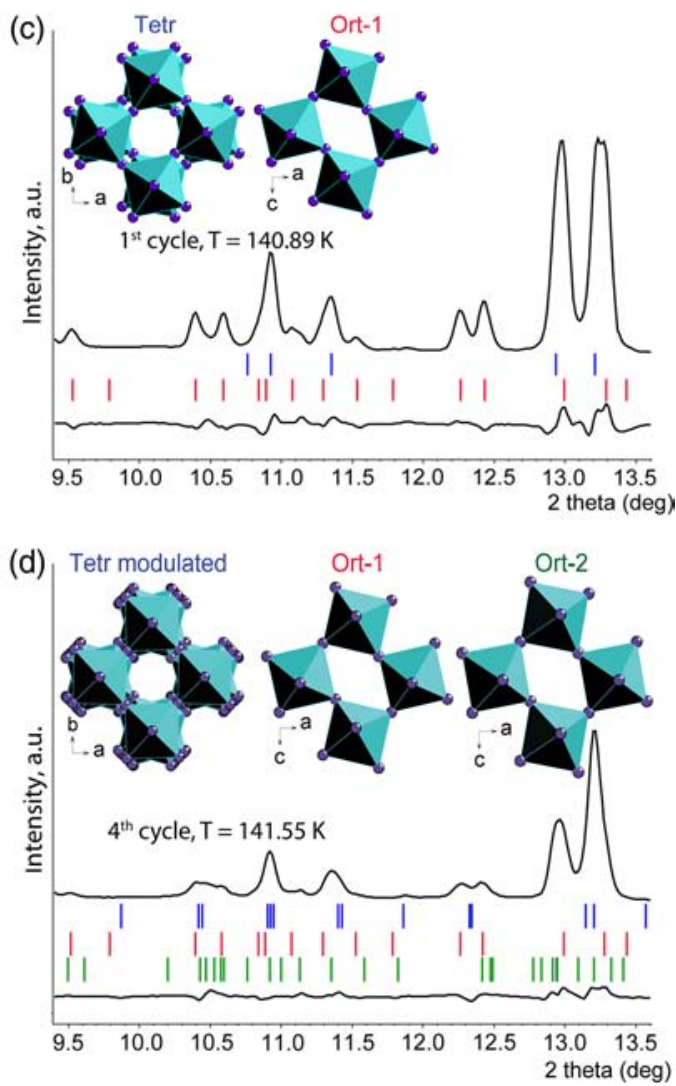

Figure 1. Temperature-dependent XRD profiles recorded upon a) cooling during the first and b) heating during the fourth thermal cycles around $160 \mathrm{~K}$. c) A part of the XRD profile for the two-phase state at $140.89 \mathrm{~K}$ in the first thermal cycle. d) A part of the XRD profile for the three-phase state at $141.55 \mathrm{~K}$ in the fourth thermal cycle (the full profile is shown in Figure S2, Supporting Information). The blue, red, and green tick-marks below the profiles mark reflections of Tetr, Ort-1, and Ort-2 phases, respectively. The black lines below the ticks refer to the difference between the observed and calculated profiles. For improved clarity of the structural diagrams, the MA cation is omitted. 
(a)

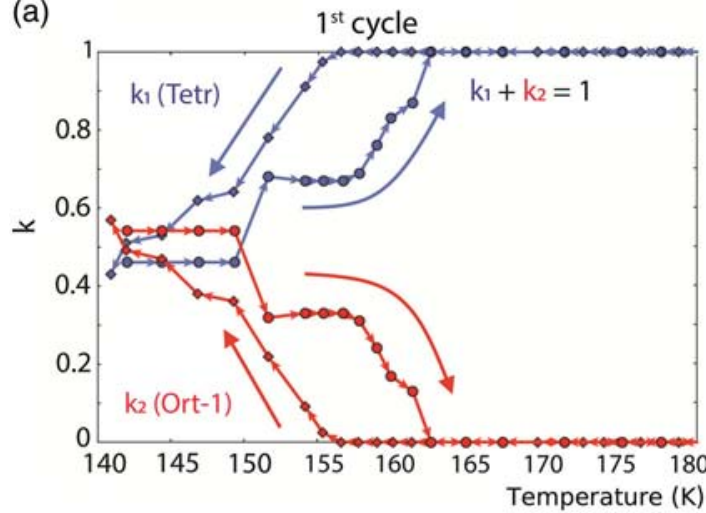

(c)

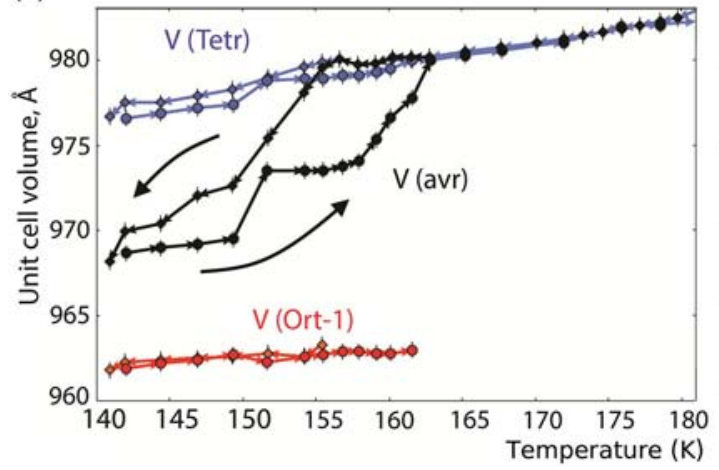

(b)

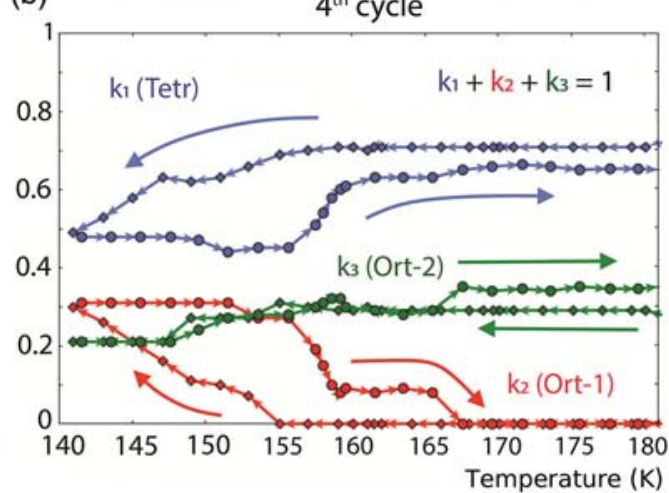

(d)

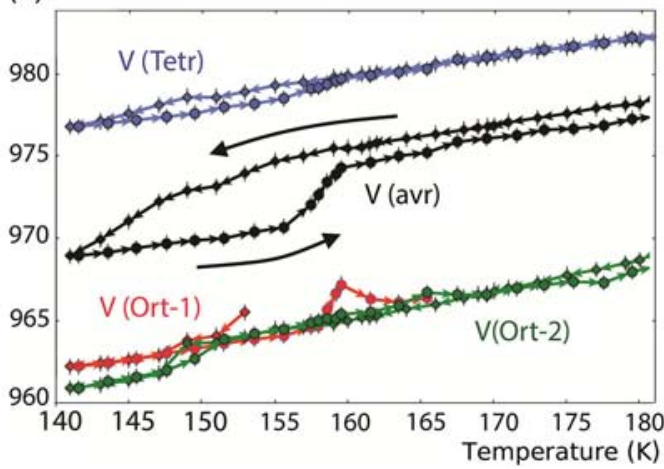

Figure 2. Temperature dependence of the phase composition and the unit cell volume of $\mathrm{MAPbl}_{3}$ in the first and fourth thermal cycles around $160 \mathrm{~K}$. $\mathrm{a}, \mathrm{b})$ The coefficients $k_{1}, k_{2}$, and $k_{3}$ define the contribution of the tetragonal (Tetr), orthorhombic (Ort-1), and boundary (Ort-2) phases at each temperature. c,d) The average unit cell volume, $V(a v r)$, was calculated using these coefficients. The error bar indicates one standard deviation for each point.

heated from 140 to $180 \mathrm{~K}$, the system returns to the single-phase state only above $164 \mathrm{~K}$, indicating a hysteresis that is consistent with a first-order phase transition.

Fourth Cycle: The XRD data plotted in Figure 1b indicate a more complex system in the fourth thermal cycle. A different orthorhombic phase, Ort-2, is now present over the full temperature range $140-180 \mathrm{~K}$. The numerous weak diffraction peaks, shown in Figure 1d, point to a modulation of the tetragonal phase, which has been accounted for in the structure refinement process (see Figure S2, Supporting Information). The modulated Tetr structure, represented in Figure 1d, accommodates a slight rotation of the $\mathrm{PbI}_{6}$ octahedra around the fourfold axis. Figure $3 \mathrm{~b}$ shows that independent of the temperature the two unit cell parameters of the Ort- $2(a \approx c)$ phase are close to the parameters of the Tetr phase $(a=b)$. This fact suggests an epitaxial intergrowth between the Tetr and Ort-2 phases. Indeed, the coefficients of the phase contribution, $k_{1} \neq 0$ for Tetr and $k_{3} \neq 0$ for Ort- 2 in Figure $2 \mathrm{~b}$, indicate the coexistence of the Ort- 2 and Tetr phases at all temperatures between 140 and $180 \mathrm{~K}$. In addition, Ort- 1 and Ort- 2 phases, as shown in Figure 1d and Figure S1, Supporting Information, are topologically similar and differ only by the orientations of the MA cations. Hence, the Ort- 2 phase can be considered as a boundary phase linking the Tetr and Ort-1 domains, as shown in Figure 4. The Rietveld structure refinements based on the powder XRD data successfully confirmed this three-phase state of $\mathrm{MAPbI}_{3}$ (Figure S2, Supporting Information).
Similarly to what was observed in the first cycle, the thermal hysteresis in the contributions of the phases Tetr $\left(k_{1}\right)$ and Ort-1 $\left(k_{2}\right)$ as shown in Figure 2b confirms the first-order nature of the phase transition. ${ }^{[22]}$ However, a comparison of Figure $2 \mathrm{c}, \mathrm{d}$ shows a significant smoothing of the hysteresis for the average unit cell volume, $V(\operatorname{avr})$, as a result of thermal cycling. This smoothing is due to the presence of the boundary phase Ort-2. It can be expected that the hysteresis of $V($ avr) would disappear with more thermal cycles. It is shown in Figure $2 \mathrm{~d}$ that the unit cell volumes of the Ort- 1 and Ort- 2 phases are practically identical, making them thermodynamically equivalent. Consequently, we expect that with thermal cycling, the Ort-1 phase could be partially or completely replaced by the Ort-2, thus bringing the system to a state of thermodynamic equilibrium characterized by the coexistence of only two mutually intergrown phases, Tetr and Ort-2.

To summarize, the structural refinement results for the thermal cycling of $\mathrm{MAPbI}_{3}$ between 140 and $180 \mathrm{~K}$ indicate that the first-order phase transition, accompanied by the coexistence of the Tetr and Ort- 1 phases, gives rise to domain formation. ${ }^{[23,24]}$ In addition, repeatedly crossing the phase transition leads to a gradual reduction and smoothing of the jump in the average unit cell volume $V($ avr), which is thermodynamically favorable. The changes throughout the thermal cycling occur due to the formation of the Ort- 2 phase-a boundary state between the Tetr and Ort-1 phases. 
(a)

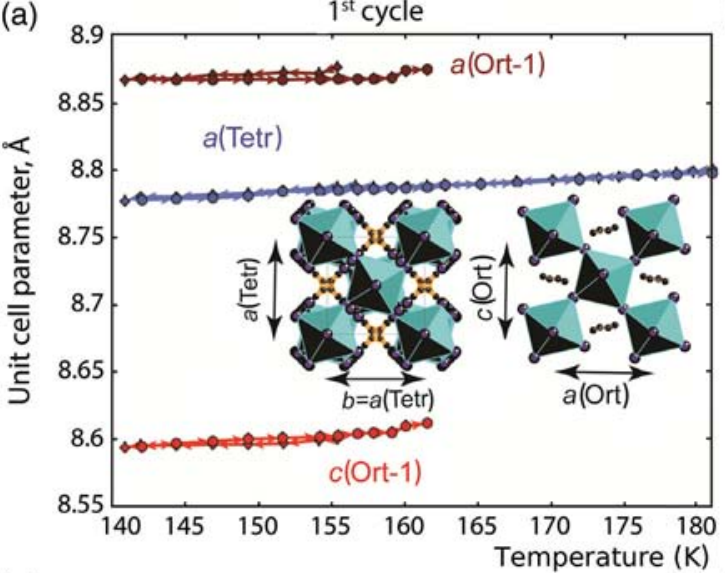

(c)

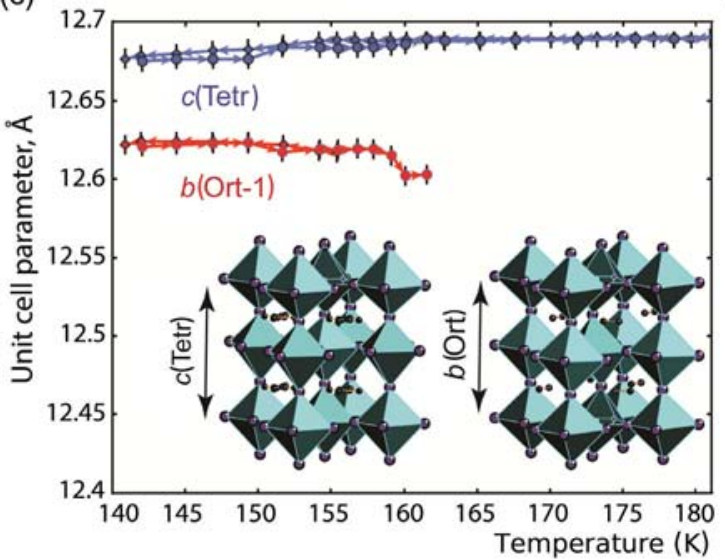

(b)

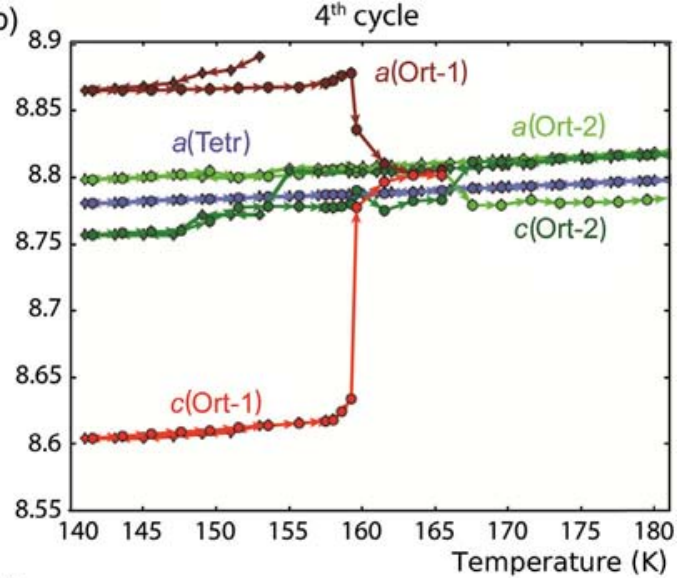

(d)

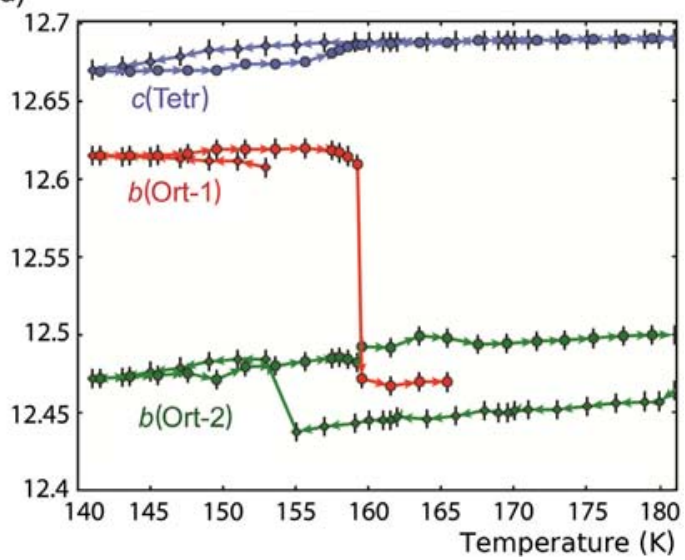

Figure 3. Temperature dependence of the unit cell parameters of the Tetr, Ort-1, and Ort-2 phases revealed in the first and fourth thermal cycle around $160 \mathrm{~K}$. The directions of the temperature sweeps are indicated by arrows between dots in the plots. The error bar indicates three standard deviations for each point in the plots.

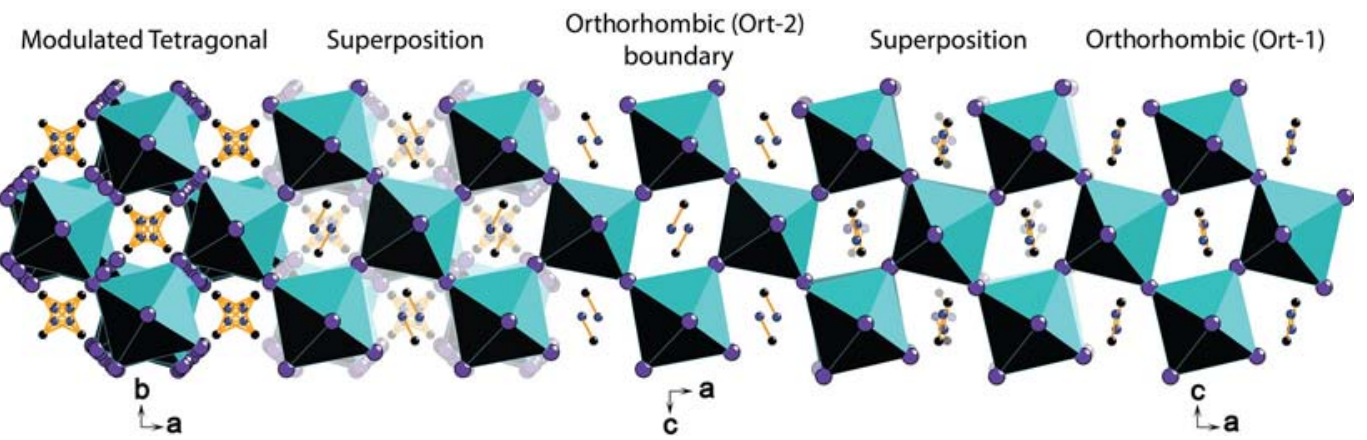

Figure 4. Stacking of the modulated tetragonal (Tetr) and orthorhombic (Ort-1) phase through a boundary phase (Ort-2) at $141.55 \mathrm{~K}$ in the fourth thermal cycle around $160 \mathrm{~K}$.

\subsubsection{Resistivity Measurements}

The contribution of the Tetr phase at the lower end of the explored temperature range $(140 \mathrm{~K})$ remains constant throughout the whole experiment (Figure 2a,b), and the boundary phase Ort-2, which is absent in the first sweep, comes from the Ort-1Ort- 2 transformation as a result of thermal cycling. Given that the contribution of Ort- 2 scales up with the number of cycles $\left(k_{3}=0\right.$ in the first thermal cycle and $k_{3}=0.25 \pm 0.05$ during the fourth thermal cycle), we expect a growth of the number of domains. This statement is supported by the electrical resistance of $\mathrm{MAPbI}_{3}$ single crystal (Figure 5), which shows at a given temperature, there is a continuous increase over the six successive thermal cycles. The complex temperature dependence of the 


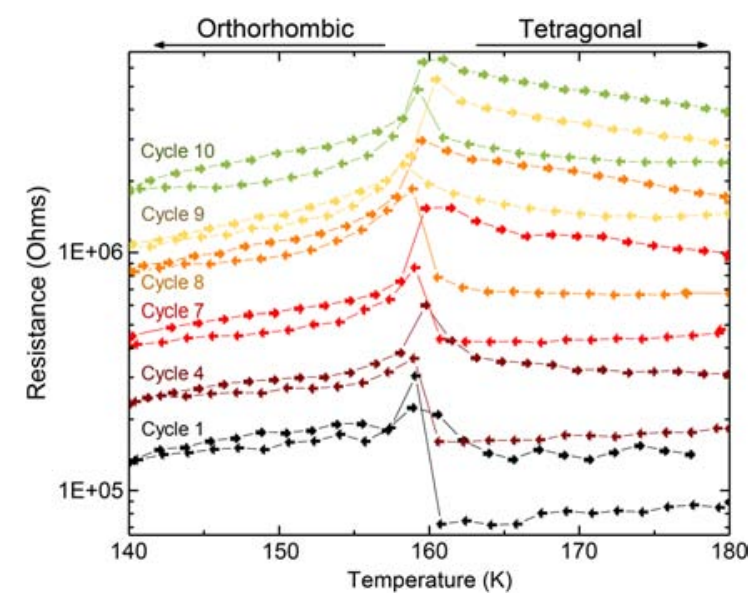

Figure 5. Temperature dependence of the electrical resistance of $\mathrm{MAPbl}_{3}$ during thermal cycling around $160 \mathrm{~K}$. The corresponding marker for each point in the plots covers experimental errors.

resistivity has previously been discussed by Pisoni et al., ${ }^{[25]}$ and the peak in the resistivity at $160 \mathrm{~K}$ is explained by the enhanced scattering of electrons due to increasing number of domain boundaries.

It has been pointed out that the efficiency of the charge carrier trapping sharply decreases in the vicinity of the boundary phase. ${ }^{[2]}$ The apparent transition temperature fluctuates slightly throughout multiple cycles because it is strongly dependent on the local properties of the material and, in particular, on the type and density of structural and chemical defects. Indeed, the Ort-1 and Tetr phases have different symmetries, and the actual symmetry of the defects can encourage the formation of either structures; hence, the transition temperature can increase or decrease.

\subsubsection{Heat Capacity Measurements}

The heat capacity data of $\mathrm{MAPbI}_{3}$ single crystal for the lowtemperature transition that are shown in Figure $\mathbf{6}$ (calculated changes in entropy are in Figure S3, Supporting Information) substantiate the XRD results and suggest a jump in the structural

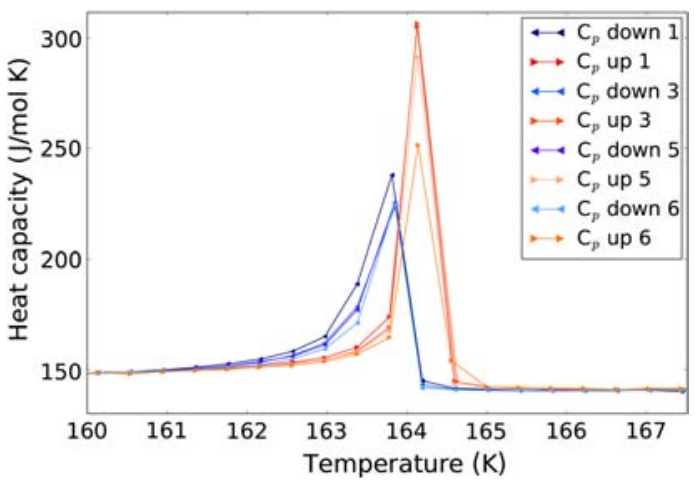

Figure 6. Temperature dependence of the heat capacity, $C_{\mathrm{p}}$, of $\mathrm{MAPbl}_{3}$ during thermal cycling around $160 \mathrm{~K}$. Cycles 2 and 4 have been omitted for clarity. The corresponding marker for each point in the plots covers experimental errors. order parameter. The structural transition is reflected in the heat capacity data as a sharp peak. In agreement with the resistivity measurements, the existence of a hysteresis indicates that the transition is first order. Also, the decrease of the heat capacity peak value with thermal cycling reflects the substitution of Ort-1 phase for Ort-2.

\subsection{The High-Temperature Tetragonal to Cubic Phase Transition}

As the crystal is warmed up toward and past room temperature, the bonding between MA and iodine in the tetragonal phase of $\mathrm{MAPbI}_{3}$ is progressively weakened. Thus, the cation starts to tumble inside the inorganic framework. ${ }^{[26]}$ The rotation angle of the octahedra in the tetragonal phase decreases monotonically with increasing temperature, and simultaneously, the lattice constants $c$ and $a$ become equal, resulting in the high-temperature cubic phase above $330 \mathrm{~K}^{[27]}$

It has been suggested that the structural change is accompanied by a ferroelectric to paraelectric transition, ${ }^{[28]}$ which is plausible, given that the tetragonal (space group I422) and cubic $(P m-3 m)$ phases, respectively, lack and possess an inversion symmetry. ${ }^{[14]}$ The high-temperature transition results in the reduction of the unit cell in the $c$-direction and could be driven by an instability of the crystal with respect to the emergence of a particular phonon mode. ${ }^{[29]}$ This hypothesis is supported by the theoretical work of Saidi and Choi, ${ }^{[30]}$ in which the stabilization of the cubic phase is explained through vibrational energy and entropy considerations.

\subsubsection{X-Ray Data}

In situ powder XRD experiments were conducted on ground $\mathrm{MAPbI}_{3}$ crystals thermally cycled six times between 310 and $350 \mathrm{~K}$ (see Experimental Section for details). The lattice structure and the unit cell parameters were refined across the explored temperature range for the first and sixth cycles. The XRD profiles shown in Figure 7a indicate a gradual transition between the tetragonal and cubic phases in the first cycle, whereas those in Figure $7 \mathrm{~b}$ imply the occurrence of an incommensurately modulated phase in the sixth cycle. We describe all these structural changes in terms of a single tetragonal $(3+3)$-dimensional superspace group generated from the space group $P 4 / \mathrm{mmm}^{[31]}$ which is applied to the pseudocubic unit cell with $a \approx c \approx 6.3 \AA$. The modulation vectors are $\mathbf{q}_{1}=\alpha \mathbf{a}^{*}+\alpha \mathbf{b}^{*}, \mathbf{q}_{2}=-\alpha \mathbf{a}^{*}+\alpha \mathbf{b} *$, and $\mathbf{q}_{3}=\gamma \mathbf{c}^{*}$, where $\alpha=\left(0.5-\Delta_{1}\right)$ and $\gamma=\left(0.5-\Delta_{2}\right)$ can be either rational if the parameters $\Delta_{1}$ and/or $\Delta_{2}$ are 0 , or irrational otherwise. If $\Delta_{1}=\Delta_{2}=0.5$, then $\alpha=\gamma=0$ and the space group describes the $3 \mathrm{D}$ pseudocubic structure. In the $a=c$ case, the structure acquires the cubic symmetry of the $P m-3 m$ supergroup. Using this structural representation, only three atoms, located in fixed positions ( $\mathrm{Pb}$ : 1a (000); I1: $1 \mathrm{~b}(001 / 2)$; I2: $2 \mathrm{f}(1 / 200))$, define the inorganic framework. The lattice parameters $a$ and $c$ were refined using only the main diffraction reflections indexed with only $a^{*}, b^{*}$, and $\mathbf{c}^{*}$ in the reciprocal lattice. Figure 8 shows these parameters as a function of temperature for the first and sixth thermal cycles. The coefficients $\alpha$ and $\gamma$ of the modulation vectors were refined using the whole set of reflections, including the 


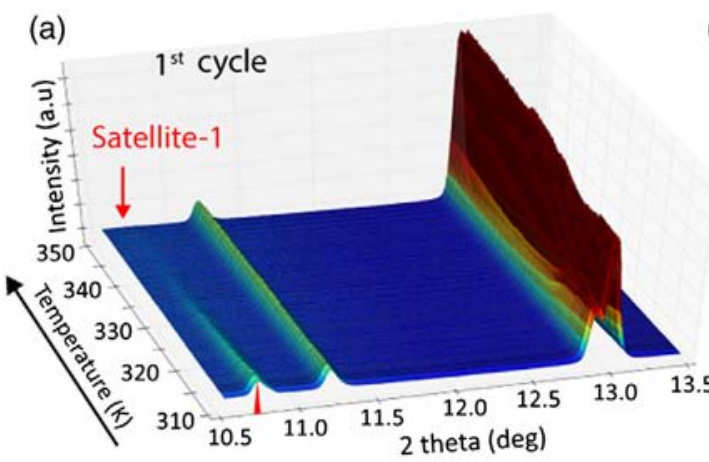

(c) $\quad 1^{\text {st }}$ cycle $\mathrm{T}=320.16 \mathrm{~K}$

(b)

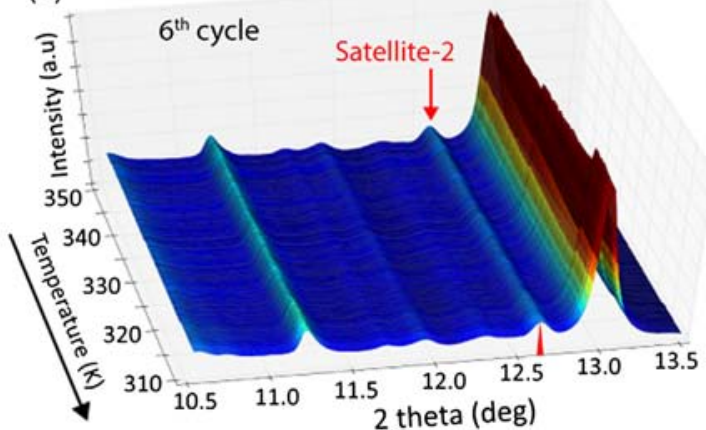

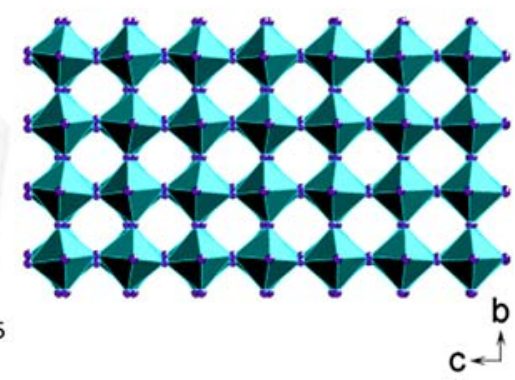

(d)

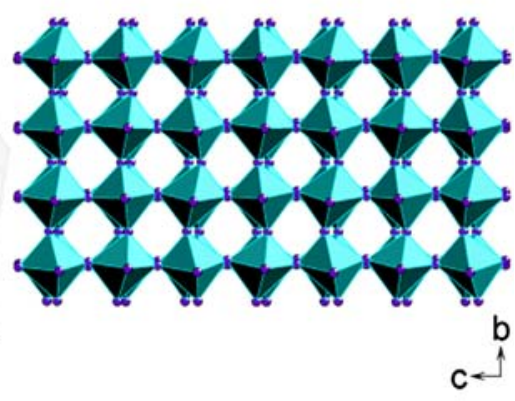

Figure 7. Temperature-dependent XRD profiles recorded upon a) heating during the first and b) cooling during the sixth thermal cycles around $330 \mathrm{~K}$. c) The $(b c)$ projection of a portion of the incommensurately modulated tetragonal structure in the first thermal cycle at $320.16 \mathrm{~K}$. d) The projection of a portion of the incommensurately modulated tetragonal structure in the sixth thermal cycle at $320.16 \mathrm{~K}$. For clarity, the MA cation is omitted.

(a)

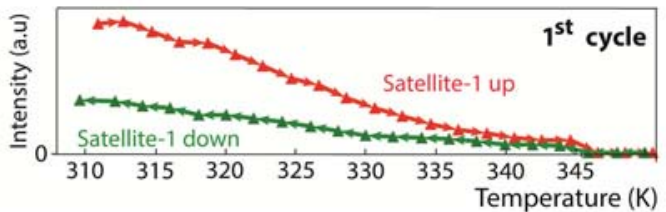

(b)

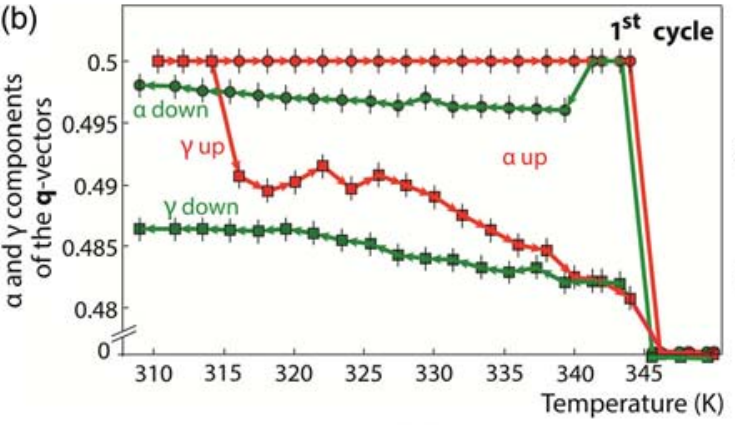

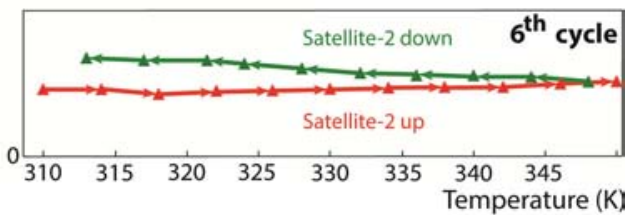

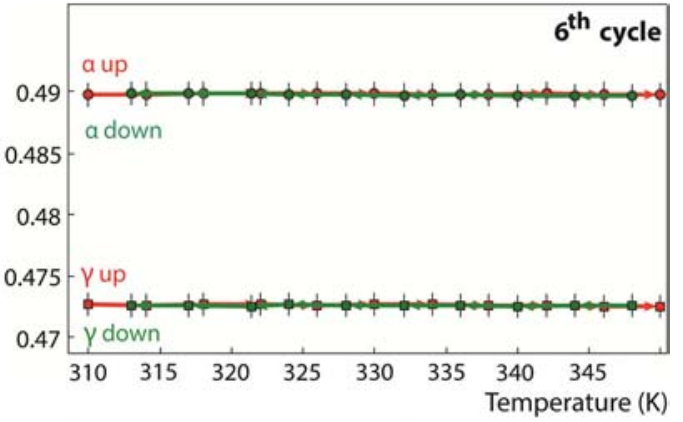

Modulation vectors: $\mathbf{q}_{\mathbf{1}}=\mathbf{a} \mathbf{a}^{*}+\mathbf{a b}^{*}, \mathbf{q}_{\mathbf{2}}=-\mathbf{a}^{*}+\mathbf{a b}^{*}$ and $\mathbf{q}_{\mathbf{3}}=\gamma$

Figure 8. Temperature dependence of the crystallographic characteristics for the incommensurately modulated structure of $\mathrm{MAPbl}_{3}$ in the first and sixth thermal cycles around $330 \mathrm{~K}$. a) The integrated intensity of the strongest satellite. b) Coefficients of the indicated modulation vectors. The error bar indicates one standard deviation for each point in the plots. In the sixth thermal cycle, the weak temperature dependence of the quantities indicates the stabilization of the modulated phase, whereas an increase in the intensity of the satellite indicates the evolution of this phase toward an amplification of displacements of atoms from their average position.

significantly weaker satellites that can be indexed only if $\alpha$ and $\gamma$ differ from 0 . Figure 8 shows the temperature dependence of their integrated intensity as well as the change in the $\alpha$ and $\gamma$ coefficients for the modulation vectors. The existence of the satellites (see also Figure S4, Supporting Information) indicates the presence of modulation waves of atomic displacements. The stronger intensities of satellites and the larger displacements are expected. Consequently, the absence of satellites denotes a 
primitive, nonmodulated structure with the unit cell parameters mentioned above and atomic positions in accordance with the tetragonal space group $P 4 / \mathrm{mmm}$. The $P m-3 m$ supergroup, which is characteristic of the cubic phase, requires identity of $a$ and $c$ lattice parameters.

First C $\gamma$ cle: On the first heating stage, in Figure 8a, the intensity of the strongest satellite decreases to zero only above $346 \mathrm{~K}$, indicating that at this temperature only the cubic phase is expected. ${ }^{[30]}$ However, in conformity with Tyson et al., ${ }^{[32]}$ we observed a pseudocubic unit cell in the whole $310-350 \mathrm{~K}$ range. Another degenerate case, when $\alpha=\gamma=0.5$, was observed below $315 \mathrm{~K}$ during the first thermal cycle heating stage. These rational values correspond to the room temperature tetragonal unit cell. Indeed, the vectors $\mathbf{q}_{1}=0.5 \mathbf{a}^{*}+0.5 \mathbf{b} *$ and $\mathbf{q}_{2}=-0.5 \mathbf{a} *+0.5 \mathbf{b} *$ define the tetragonal basis of the cell as $\mathbf{a}_{\text {tetr }}=\mathbf{a}+\mathbf{b}$ and $\mathbf{b}_{\text {tetr }}=-\mathbf{a}+\mathbf{b}\left(a_{\text {tetr }}=b_{\text {tetr }}=\right.$ $a \sqrt{ } 2 \approx 8.9 \AA)$, and the vector $\mathbf{q}_{3}=0.5 \mathrm{c} *$ defines the doubling of the $c$ parameter, $\mathbf{c}_{\text {tetr }}=2 \mathrm{c}\left(c_{\text {tetr }}=2 c \approx 12.6 \AA\right)$.

The clear changes in the unit cell parameters, modulation vectors, and intensity of the strongest satellite reflections indicate that the structural transformation from the room temperature tetragonal phase directly into the pseudocubic phase occurs only in the first thermal cycle. In accordance with a previous report, ${ }^{[32]}$ we confirm the presence of an intermediate phase, in the temperature range of 315-346 K between tetragonal and cubic phases.

(a)

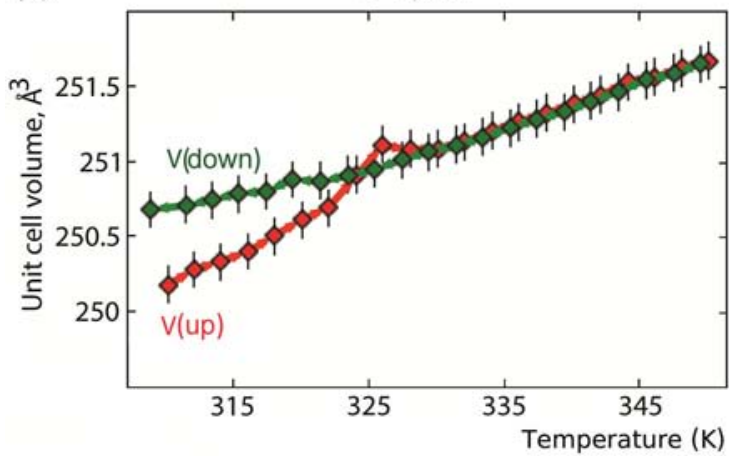

(c)

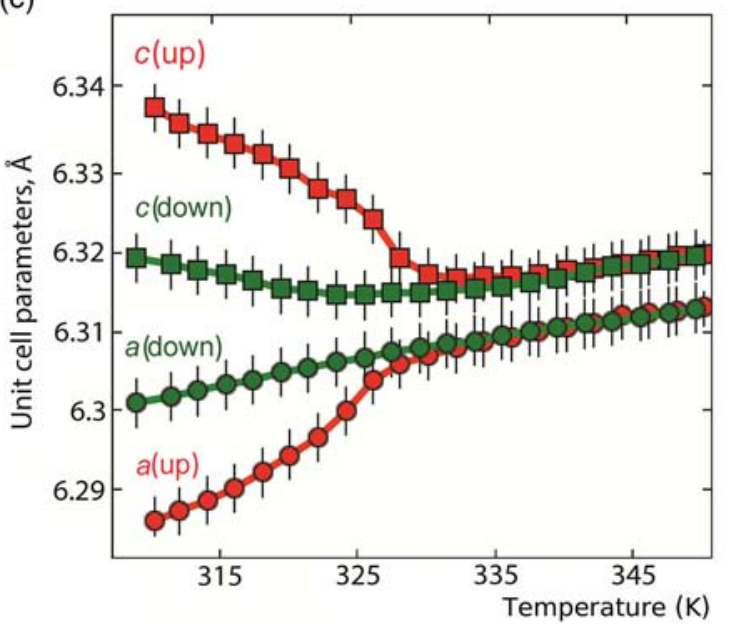

The crystal structure of the intermediate phase has been refined by the Rietveld method for 320.16 K (see Figure S5, Supporting Information). It is primarily the I atoms that are displaced due to the structural modulations. A portion of the structure shown in Figure 7c shows that these displacements result in a wave of tilted $\mathrm{PbI}_{6}$ octahedra around the $a$ and $b$ axes, whereas one would rather expect a rotation around the $c$-axis, which is typical for the tetragonal phase. This "tilt wave" results in the crystal spatially alternating between the two types of regions: without (pseudocubic) and with periodically modulated tilt of the octahedra along the $c$-axis (see Figure S6, Supporting Information). Figure $8 \mathrm{a}$ shows that the intensity of the strongest satellite continuously decreases with increasing temperature and is completely suppressed when the pseudocubic phase sets in. This behavior indicates that the average size of the pseudocubic region grows, and the mean magnitude of the displacements of I atoms becomes smaller.

Sixth Cycle: In the sixth thermal cycle, we find the presence of a single stable phase. It is characterized by the irrational temperature-independent modulation coefficients $\gamma \approx 0.473$ and $\alpha \approx 0.490$ (Figure 8 ), which point to an aperiodicity of the crystal along the $c$-axis as well as in the $a b$ plane. The unit cell parameters change linearly with temperature, as shown in Figure 9. Their values for both the warm-up and cool-down are now

(b)

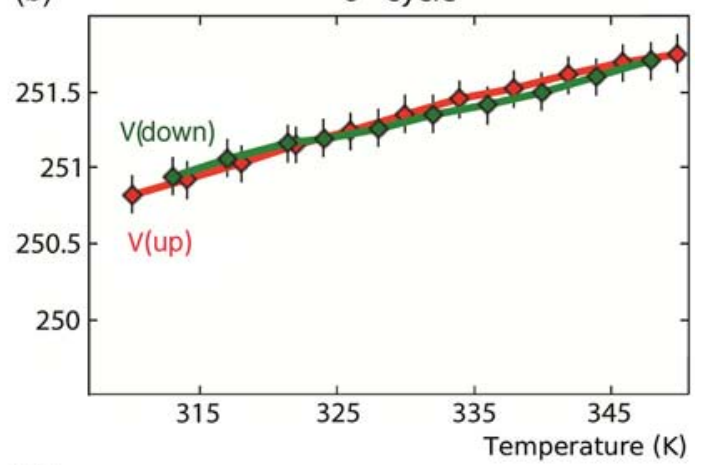

(d)

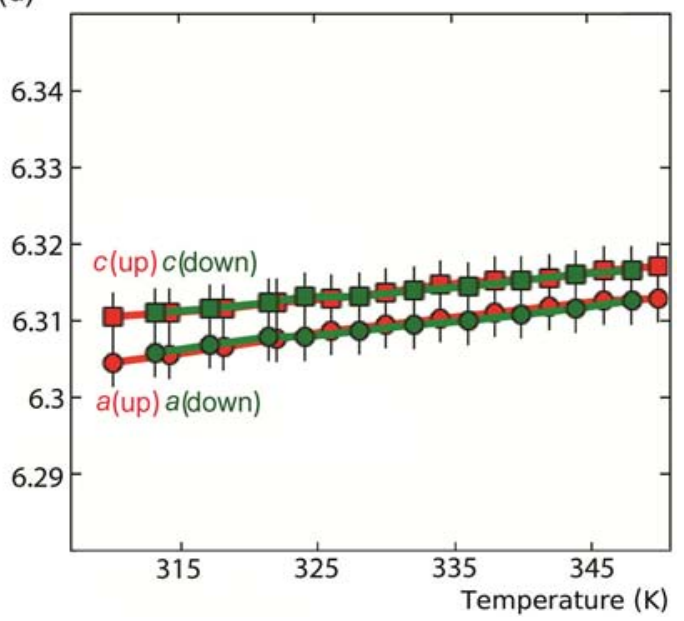

Figure 9. Temperature-dependent variation of $a, b)$ the tetragonal unit cell volume and $c, d$ ) its $a$ and $c$ parameters in the first and sixth thermal cycle around $330 \mathrm{~K}$. The error bar indicates one standard deviation for each point in the plots. In the sixth thermal cycle, the phase has been stabilized with respect to the plotted parameters. 
identical, suggesting that the system has settled into a thermodynamically stable state.

A crystal structure estimation for the phases present in the sixth thermal cycle at 350.2, 321.3, and 310.2 K (Rietveld refinements in Figure S7, Supporting Information) shows that the "tilt wave" occurs along lattice axes in agreement with irrational values the of $\alpha$ and $\gamma$ components in the modulation vectors (Figure 8). Figure 7d shows the variation of the tilt of the octahedral. Despite the fact that each octahedron is tilted, the degree of tilt varies within a coherent area. Less tilted octahedra form pseudocubic structure regions, which smoothly turn into tetragonal, consisting of more tilted octahedra. With such a change from one to another unit cell, only an approximate boundary between these regions and, accordingly, an approximate size of the pseudocubic regions can be discussed. Due to incommensurability in three axes (three independent q-vectors of modulation), even the approximate size of the regions is varied depending on a portion taken from the aperiodic structure. However, the reconstruction of an identical portion of the structure taken at different temperatures can illustrate the temperature dependence of the size. Such illustration and details of the reconstruction are given in Supporting information (Figure S8, Supporting Information) for a structure portion of $14 a \times 14 b \times 5 c(10 \mathrm{~nm} \times 10 \mathrm{~nm} \times 3 \mathrm{~nm})$. In the selected area, the approximate size of pseudocubic regions increases with increasing temperature from 310.2 to $350.2 \mathrm{~K}$, indicating a transformation from "mainly tetragonal" to "mainly pseudocubic."

Any dynamic distortion in a crystal can be described in terms of a complete set of normal vibrational modes. When a solid undergoes a structural phase transition, the process is often described in terms of anomalous behavior of a single mode, characterized by its displacement eigenvector, frequency, and wavelength. If the space group of one phase is a subgroup of that of the other phase, the transition may proceed via a continuous distortion of the unit cell, i.e., the transition is of second order. ${ }^{[33]}$ Within this framework, as the high-temperature pseudocubic phase is approached, the frequency of a particular low-energy phonon mode grows. In Figure S9, Supporting Information, one can see the increase in diffuse scattering in the diffraction pattern, which is related to an enhancement of the vibrational energy of the lattice. The existence of transverse and longitudinal acoustic phonon modes as well as one transverse optical branch in the inorganic framework at $350 \mathrm{~K}$ has been shown by Beecher et al. ${ }^{[34]}$ Due to the low-energy modes associated with the inorganic lattice, phonons provide a major contribution to stabilize the cubic phase at a high temperature. From the structural refinement, we see the "tilt wave;" however, a proper mapping of the phonon modes to the structure modulations will require an additional study.

To summarize, the structural refinements for the thermal cycling around the high-temperature tetragonal-cubic phase transition show that the sharp change of the unit cell parameters at $330 \mathrm{~K}$ occurs only in the first thermal cycle. During the same initial pair of temperature sweeps, the change in the mobility of the $\mathrm{PbI}_{6}$ octahedra from rotation to tilt relative to the tetragonal $\mathrm{c}$-axis underlies the $315 \mathrm{~K}$ second-order phase transition, and the first-order phase transition accompanied by a $(3+3)$ dimensional symmetry transformation into a $3 \mathrm{D}$ symmetry is observed at $345 \mathrm{~K}$. Thermal cycling results in the occurrence of a $(3+3)$-dimensional incommensurately modulated phase, which consists of pseudocubic and pseudotetragonal regions as a result of the "tilt-wave" modulation of the octahedra orientations.

\subsubsection{Resistivity Measurements}

To explore the effect of thermal cycling around the hightemperature transition on the electronic properties of $\mathrm{MAPbI}_{3}$, we measured the resistance of a single crystal between 310 and $350 \mathrm{~K}$. It can be seen from Figure 10 that in contrast to what was observed around the low-temperature transition, here the resistivity peak, just below $330 \mathrm{~K}$, disappears after multiple thermal cycles. This supports the detected structural transformations. Indeed, the change in sample resistance observed between the third and fifth cycles can be attributed to the stabilization of the incommensurately modulated phase that contains both the cubic-like and the tetragonal-like regions. The more progressive variation in the sample resistance during the fifth and seventh thermal cycles can be associated with the evolution of the incommensurate phase, which is inferred from the increased intensity of satellite reflections presented in Figure 8a (see also Figure S4, Supporting Information).

\subsubsection{Heat Capacity Measurements}

Both the high- and low-temperature phase transitions of $\mathrm{MAPbI}_{3}$ are technically of first order; however, the high-temperature transition is close to being of second order. ${ }^{[35]}$ In addition to the fact that both the tetragonal and the pseudocubic phases can be described by one superspace group, the heat capacity data shown in Figure 11 (which is consistent with those in the literature ${ }^{[32]}$ ) support this statement. There is no apparent hysteresis, and the broad peak more closely resembles a power law divergence rather than a delta function. Changes in entropy $(\Delta S)$ are shown in

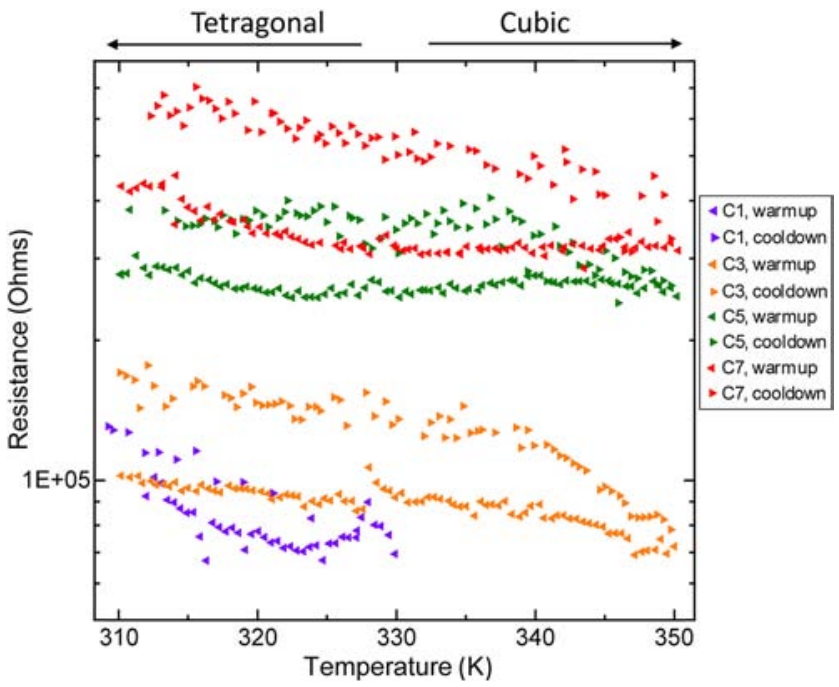

Figure 10. Temperature dependence of the electrical resistance of $\mathrm{MAPbl}_{3}$ during thermal cycling around $330 \mathrm{~K}$. Cycles 2, 4, and 5 have been omitted for clarity. The corresponding marker for each point in the plots covers experimental errors. 


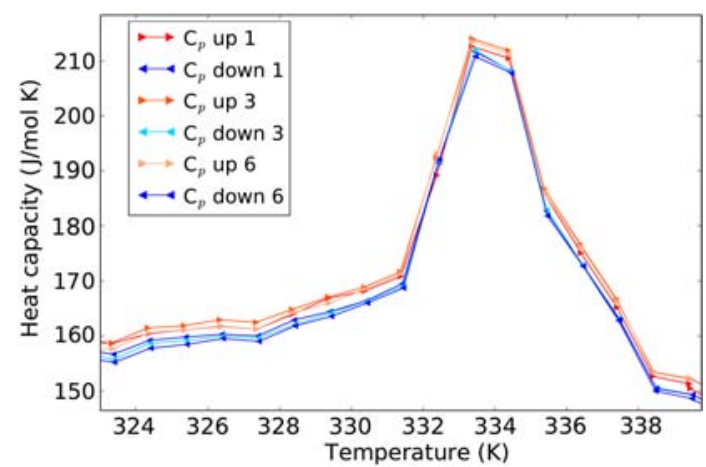

Figure 11. Temperature dependence of the heat capacity, $C_{\mathrm{p}}$, of $\mathrm{MAPbl}_{3}$ during thermal cycling around $330 \mathrm{~K}$. Cycles 2, 4, and 5 have been omitted for clarity. The corresponding marker for each point in the plots covers experimental errors.

Figure S10, Supporting Information. The jump in entropy is smoother for the high-temperature transition than for the lowtemperature one (Figure S3, Supporting Information). What stands out from the data in Figure 11 is the fact that the heat capacity value is higher below the transition than above it. Onoda-Yamamuro et al. ${ }^{[35]}$ attributed an excess heat capacity in the region below the transition to the associated latent heat smeared out toward the low-temperature side. Moreover, the results indicate that the phase transition exists even after six thermal cycles.

The structural characteristics of the crystal can explain the behavior of the heat capacity during the thermal cycling. At the start of the first cycle, the strongest diffraction peak indicating the tetragonal phase (Satellite-1 in Figure 7a) continuously vanishes with increasing temperature. The highest rate of this disappearance (Figure 8a) coincides with the sharp rise in heat capacity (Figure 11) around $330 \mathrm{~K}$. Starting from $335 \mathrm{~K}$, both the intensity and the heat capacity value fall more smoothly. Therefore, the observed changes in the heat capacity are attributed to the cubic-tetragonal transition. This transformation is clearly a characteristic of the first thermal cycle. As discussed in Section 2.2.1 (see also Figure S8, Supporting Information), a similar transformation is observed in the sixth thermal cycle, however, within the evolution of a single incommensurately modulated phase from "mainly cubic" to "mainly tetragonal."

\section{Conclusions}

We investigated how thermal cycling through the low- and hightemperature structural transitions influences the crystal structure and electronic properties of $\mathrm{MAPbI}_{3}$ crystals. XRD, electrical resistivity, and heat capacity measurements were performed at temperatures covering each phase transition. At low temperature, repeatedly crossing the tetragonal-orthorhombic phase transition $(160 \mathrm{~K})$ generates an increase in the concentration of the domains of different phases. After four cycles, the new phase, Ort-2, is present above and below the transition and acts as a boundary phase linking the Tetr and Ort-1 domains. At high temperature, while cycling through the tetragonal-cubic phase transition $(330 \mathrm{~K})$, we observed the emergence of a single phase that consists of pseudocubic and pseudotetragonal regions with incommensurate modulations of the $\mathrm{PbI}_{3}$ octahedra tilt. We describe a series of phase transformations within the single $(3+3)$-dimension superspace group generated from the space group $\mathrm{P} 4 / \mathrm{mmm}$ and applied to the pseudocubic tetragonal unit cell with $a \approx c \approx 6.3 \AA$. In addition, the coefficients $\alpha$ and $\gamma$ of the modulation vectors $\mathbf{q}_{1}=\alpha \mathbf{a}^{*}+\alpha \mathbf{b}^{*}, \mathbf{q}_{2}=-\alpha \mathbf{a}^{*}+\alpha \mathbf{b}^{*}$, and $\mathbf{q}_{3}=\gamma \mathbf{c}^{*}$ determine the temperature- and the cycling-dependent state of the structure. Within our interpretation, the interaction between the organic and inorganic parts of the structure is at the core of the phase transitions. Thus, it may be possible to improve the stability of the crystal through cation substitution ${ }^{[36]}$ (at the risk of modifying the electronic properties of the material). Alternatively, particular phases can be stabilized in the desired temperature range by means of atomic substitution or with the introduction of inert gas into the lattice. ${ }^{[37]}$

\section{Experimental Section}

Synthesis: $\mathrm{MAPbl}_{3}$ was synthesized using a slightly modified version ${ }^{[17,38]}$ of the method described by Poglitsch and Weber. ${ }^{[39]}$ The single crystals were prepared by precipitation from a concentrated aqueous solution of hydriodic acid (57 wt\% in $\mathrm{H}_{2} \mathrm{O}, 99.99 \%$ Sigma-Aldrich) containing lead (II) acetate trihydrate (99.999\%, Acros Organics) and the respective amount of $\mathrm{CH}_{3} \mathrm{NH}_{2}$ solution ( 30 wt\% in $\mathrm{H}_{2} \mathrm{O}$, Sigma-Aldrich). A constant $55-42^{\circ} \mathrm{C}$ temperature gradient was applied to induce a continuous precipitation of the solute. Large $(1 \times 2 \mathrm{~mm})$ silver-gray $\mathrm{MAPbl}_{3}$ crystals were grown within 2 days. To prevent any reaction with moisture, the synthesized crystals were immediately transferred and kept in a desiccator prior to the measurements.

$X$-Ray Data: $\mathrm{MAPbl}_{3}$ crystals were ground just before the experiment in a mortar and loaded into a quartz capillary. The synchrotron radiation powder XRD data were collected as a function of temperature using a wavelength $\lambda=0.7129 \AA$ and $5 \mathrm{~s}$ of exposure time with the PILATUS@SNBL detector at the Swiss-Norwegian Beam Lines, European Synchrotron Radiation Facility. ${ }^{[40]}$ The temperature sweeps across the transitions temperature took place at $0.7 \mathrm{~K} \mathrm{~min}^{-1}$ in both directions using an Oxford Cryostream $700+$ nitrogen blower. The powder diffraction data were processed with BUBBLE software. ${ }^{[40]}$ Crys-AlisPro software was used for the processing of the experimental data. ${ }^{[4]]}$ Structural calculations were made with the JANA2006 software. ${ }^{[42]}$ Further structural and experimental information is available as a CIF file in the Supporting Information.

Resistivity Measurements: Electrical resistivity data as a function of temperature were obtained in helium atmosphere using a standard four-point probe direct current (DC) method with a Keithley 220 current source and a Nanovoltmeter 2182. Gold wires were glued on the sample with carbon-containing paste to minimize the Schottky barrier at the interface. ${ }^{[43]}$ The $\mathrm{MAPbl}_{3}$ single crystal was illuminated by a red light $(660 \mathrm{~nm})$ produced by an ADL-66505TL AlGalnP visible laser diode and delivered to the sample via a $2 \mathrm{~mm}$ diameter polymer optical fiber (PMMA) optical fiber. The schematic diagram of the setup is shown in Figure S11, Supporting Information. The current polarity was periodically alternated to avoid the effects of charge accumulation.

Heat Capacity: Measurements of heat capacity at a constant pressure, $C_{p}$, were carried out on single crystals of $\mathrm{MAPbl}_{3}$ using the Quantum Design PPMS (Physical Property Measurement System) heat capacity option with the thermal relaxation technique.

CCDC numbers 1869093-1869096 contain the supplementary crystallographic data for this article. These data can be obtained free of charge from The Cambridge Crystallographic Data Centre via www.ccdc.cam.ac. uk/data_request/cif.

CCDC 1869093 corresponds to the incommensurately modulated state of $\mathrm{CH}_{3} \mathrm{NH}_{3} \mathrm{Pbl}_{3}$ in the first thermal cycle around $330 \mathrm{~K}$ at $320.16 \mathrm{~K}$. CCDC numbers 1869094-1869096 correspond to the three-phase state at 
$141.55 \mathrm{~K}$ in the fourth thermal cycle around $160 \mathrm{~K}$ : 1869094 - the tetragonal modulated phase; 1869095-the orthorhombic phase Ort-1; 1869096 - the boundary orthorhombic phase Ort-2.

\section{Supporting Information}

Supporting Information is available from the Wiley Online Library or from the author.

\section{Acknowledgements}

The authors are grateful for the financial support of the ERC Advanced grant Picoprop (Project Number: 670918). The authors like to thank BM01 staff for the support and help with the XRD experiments. Allocation of the beamtime at the Swiss-Norwegian Beam Lines (SNBL) by SNX council is greatly appreciated.

\section{Conflict of Interest}

The authors declare no conflict of interest.

\section{Keywords}

methylammonium lead iodide, organometallic perovskites, phase transitions, temperature cycling

Received: January 29, 2019

Revised: April 12, 2019

Published online: May 6, 2019

[1] Q. Dong, Y. Fang, Y. Shao, P. Mulligan, J. Qiu, L. Cao, J. Huang, Science, 2015, 347, 6225, 967.

[2] J.-Y. Jeng, Y.-F. Chiang, M.-H. Lee, S.-R. Peng, T.-F. Guo, P. Chen, T.-C. Wen, Adv. Mater. 2013, 25, 3727.

[3] M. M. Lee, J. Teuscher, T. Miyasaka, T. N. Murakami, H. J. Snaith, Science 2012, 338, 643.

[4] K. Mantulnikovs, A. Glushkova, P. Matus, L. Ćirić, M. Kollár, L. Forró, E. Horváth, A. Sienkiewicz, ACS Photonics 2018, 5, 1476.

[5] R. Dong, Y. Fang, J. Chae, J. Dai, Z. Xiao, Q. Dong, Y. Yuan, A. Centrone, X. C. Zeng, J. Huang, Adv. Mater. 2015, 27, 1912.

[6] B. Náfrádi, G. Náfrádi, L. Forró, E. Horváth, J. Phys. Chem. C 2015, 119, 25204.

[7] L. Gil-Escrig, G. Longo, A. Pertegás, C. Roldán-Carmona, A. Soriano, M. Sessolo, H. J. Bolink, Chem. Commun. 2015, 51, 569.

[8] W. S. Yang, B. W. Park, E. H. Jung, N. J. Jeon, Y. C. Kim, D. U. Lee, S. S. Shin, J. Seo, E. K. Kim, J. H. Noh, S. I. Seok, Science 2017, 356, 1376.

[9] NREL, Best research cell efficiencies, https://www.nrel.gov/pv/ assets/images/efficiency-chart.png (accessed: May 2015).

[10] A. Kojima, K. Teshima, Y. Shirai, T. Miyasaka, J. Am. Chem. Soc. 2009, $131,6050$.

[11] H. S. Kim, C. R. Lee, J. H. Im, K. B. Lee, T. Moehl, A. Marchioro, S. J. Moon, R. Humphry-Baker, J. H. Yum, J. E. Moser, M. Grätzel, Sci. Rep. 2012, 2, 591.

[12] B. Conings, J. Drijkoningen, N. Gauquelin, A. Babayigit, J. D'Haen, L. D'Olieslaeger, A. Ethirajan, J. Verbeeck, J. Manca, E. Mosconi, F. D. Angelis, Adv. Energy Mater. 2015, 5, 1500477.

[13] W. L. Leong, Z. E. Ooi, D. Sabba, C. Yi, S. M. Zakeeruddin, M. Grätzel, J. M., Gordon, E. A. Katz, N. Mathews, Adv. Mater. 2016, 28, 2439
[14] A. Arakcheeva, D. Chernyshov, M. Spina, L. Forró, E. Horváth, Acta Crystallogr. Sect. B: Struct. Sci. Crystal Eng. Mater. 2016, 72, 716.

[15] M. T. Weller, O. J. Weber, P. F. Henry, A. M. Di Pumpo, T. C. Hansen, Chem. Commun. 2015, 51, 4180.

[16] C. Motta, F. El-Mellouhi, S. Kais, N. Tabet, F. Alharbi, S. Sanvito, Nat. Commun. 2015, 6, 7026.

[17] A. Pisoni, J. Jacimovic, O. S. Barisic, M. Spina, R. Gaál, L. Forró, E. Horváth, J. Phys. Chem. Lett. 2014, 5, 2488.

[18] E. M. Hutter, M. C. Gélvez-Rueda, A. Osherov, V. Bulović, F. C. Grozema, S. D. Stranks, T. J. Savenije, Nat. Mater. 2017, 16, 115.

[19] H. Wang, L. Whittaker-Brooks, G. R. Fleming, J. Phys. Chem. C 2015, $119,19590$.

[20] W. Huang, S. Yue, Y. Liu, L. Zhu, P. Jin, Q. Wu, Y. Zhang, Y. Chen, K. Liu, P. Liang, S. Qu, ACS Photonics 2018, 5, 1583.

[21] C. Wehrenfennig, M. Liu, H. J. Snaith, M. B. Johnston, L. M. Herz, APL Mater. 2014, 2, 081513.

[22] A. Osherov, E. M. Hutter, K. Galkowski, R. Brenes, D. K. Maude, R. J. Nicholas, P. Plochocka, V. Bulović, T. J. Savenije, S. D. Stranks, Adv. Mater. 2016, 28, 10757.

[23] A. Dobrovolsky, A. Merdasa, E. L. Unger, A. Yartsev, I. G. Scheblykin, Nat. Commun. 2017, 8, 34.

[24] F. Brivio, J. M. Frost, J. M. Skelton, A. J. Jackson, O. J. Weber, M. T. Weller, A. R. Goni, A. M. Leguy, P. R. Barnes, A. Walsh, Phys. Rev. B 2015, 92, 144308.

[25] A. Pisoni, J. Jacimovic, B. Náfrádi, P. Szirmai, M. Spina, R. Gaál, K. Holczer, E. Tutis, L. Forró, E. Horváth, 2016, arXiv preprint arXiv: 1604.05637.

[26] A. A. Bakulin, O. Selig, H. J. Bakker, Y. L. Rezus, C. Müller, T. Glaser, R. Lovrincic, Z. Sun, Z. Chen, A. Walsh, J. M. Frost, J. Phys. Chem. Lett. 2015, 6, 3663.

[27] M. N. F. Hoque, N. Islam, Z. Li, G. Ren, K. Zhu, Z. Fan, ChemSusChem 2016, 9, 2692.

[28] Y. Kutes, L. Ye, Y. Zhou, S. Pang, B. D. Huey, N. P. Padture, J Phys. Chem. Lett. 2014, 5, 3335.

[29] S. Sugawara, T. Sato, T. Takahashi, T. Isobe, A. Nakajima, S. Matsushita, Mater. Res. Innovations 2019, 23, 49.

[30] W. A. Saidi, J. J. Choi, J. Chem. Phys. 2016, 145, 144702.

[31] S. van Smaalen, B. J. Campbell, H. T. Stokes, Acta Crystallogr. Sect. A: Found. Crystallogr. 2013, 69, 75.

[32] T. A. Tyson, W. Gao, Y. S. Chen, S. Ghose, Y. Yan, Sci. Rep. 2017, 7, 9401.

[33] P. A. Fleury, Ann. Rev. Mater. Sci. 1976, 6, 157.

[34] A. N. Beecher, O. E. Semonin, J. M. Skelton, J. M. Frost, M. W. Terban, H. Zhai, A. Alatas, J. S. Owen, A. Walsh, S. J. Billinge, ACS Energy Lett. 2016, 1, 880.

[35] N. Onoda-Yamamuro, T. Matsuo, H. Suga, J. Phys. Chem. Solids 1990, $51,1383$.

[36] Z. Li, M. Yang, J. S. Park, S. H. Wei, J. J. Berry, K. Zhu, Chem. Mater. 2015, 28, 284

[37] A. Arakcheeva, V. Svitlyk, M. Kollár, B. Náfrádi, L. Forró, E. Horváth, Found. Crystallogr. 2017, 70, C1416.

[38] E. Horváth, M. Spina, Z. Szekrényes, K. Kamarás, R. Gaal, D. Gachet, L. Forró, Nano Lett. 2014, 14, 6761.

[39] A. Poglitsch, D. Weber, J. Chem. Phys. 1987, 87, 6373.

[40] V. Dyadkin, P. Pattison, V. Dmitriev, D. Chernyshov, J. Synchrotron Radiat. 2016, 23, 825 .

[41] Oxford Diffraction, CrysAlisPRO. Agilent Technologies, 2014, Version 1.171.37.35 (release 13-08-2014 CrysAlis171.NET) compiled Aug 13 2014,18:06:01.

[42] V. Petříček, M. Dusek, L. Palatinus, Z. Kristallogr. 2014, 229, 345.

[43] X. Mettan, R. Pisoni, P. Matus, A. Pisoni, J. Jaćimović, B. Náfrádi, M. Spina, D. Pavuna, L. Forró, L., E. Horváth, J. Phys. Chem. C 2015, 119, 11506. 